\title{
Benchmarking studies of the MPISI Material Science Diffractometer at SAFARI-1
}

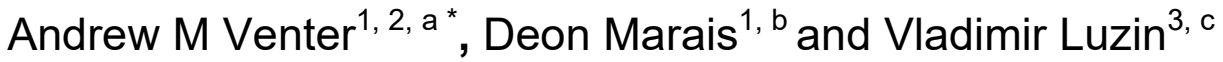 \\ ${ }^{1}$ Research and Development Division, Necsa SOC Limited, Pretoria, South Africa \\ ${ }^{2}$ DST-NRF Centre of Excellence in Strong Materials, South Africa \\ ${ }^{3}$ ANSTO, Bragg Institute, Lucas Heights, NSW, Australia \\ a Andrew.Venter@necsa.co.za, ${ }^{b}$ Deon.Marais@necsa.co.za, ${ }^{c}$ Vladimir.Luzin@ansto.gov.au
}

\section{Keywords: Neutron Strain Scanner, Benchmark, Laser Shock Peening}

\begin{abstract}
The measurement performance of the new material science instrument MPISI at the SAFARI-1 research reactor in South Africa has been comprehensively benchmarked for strain scanning applications. In addition to the traditional VAMAS Ring and Plug specimen, the benchmarking was extended to a project that required sub-millimeter spatial resolution. For the latter the MPISI performance has been compared with experimental investigations on the same sample set at the KOWARI instrument at ANSTO. Overall, good data quality and agreement have been observed between the two instruments.
\end{abstract}

\section{Introduction}

A new material science neutron diffractometer named MPISI (Materials Probe for Internal Strain Investigations; Zulu name for spotted hyena) has recently been established at the SAFARI-1 research reactor in South Africa. As part of the commissioning program the well characterized VAMAS Ring and Plug specimen [1] was investigated to confirm the general instrumental performance in terms of attainable strains and positional resolution.

Various projects have since been performed on MPISI with residual stress investigations in standard geometries using fully embedded and large gauge volumes. Resolving steep stress gradients close to surfaces however require higher precision setups and measurement strategies to achieve the desired positional accuracies. This entails positioning the apertures as close as possible to the sample, in conjunction with very accurate directional alignment to the instrument center of rotation (CoR) to ensure precise definition of the measurement gauge volume. In addition the sample reference point should be determined to within $10 \%$ of the minimum dimension of the gauge volume since poorer accuracy may lead to significant spurious strains in data points treated for partially submerged gauge volumes.

By performing comparative studies on the same samples the correspondence between the data quality, stress resolving ability and overall instrument performance can be determined.

\section{Experiment design}

To assess our high-spatial resolution measurement strategy, the depth dependences of the in-plane stresses were determined in thin laser-shock peened (LSP) aluminium plates. LSP is a new laser surface treatment technique that can introduce compressive residual stresses extending beyond $1 \mathrm{~mm}$ in depth into metallic components to enhance fatigue life and stress corrosion performance [2]. Investigations were done on the MPISI instrument using the smallest practically achievable gauge volume that was selected as a trade-off between the strain positional resolution and measurement times. The investigations were subsequently extended to the KOWARI neutron strain scanner [3] at ANSTO which is a well-established high performing instrument that, with its higher neutron flux, enabled achieving even smaller through-depth spatial resolution. 
Sample description. Four AA6056-T4 aluminium plate samples treated to different laser-shock peen parameters have been investigated on both the MPISI and KOWARI neutron strain scanners. The plate sizes were $200 \mathrm{~mm}$ (along the rolling direction) x $50 \mathrm{~mm} \times 3.2 \mathrm{~mm}$ where the top surface of each sample was laser-shock peened over an area of $25 \times 25 \mathrm{~mm}^{2}$. The LSP treatment was performed at the CSIR National Laser Centre (South Africa) using a Quanta Ray Pro-270 laser system from Spectra Physics. This was accomplished without a sacrificial ablative coating [4] using a laser power intensity of $3 \mathrm{GW} / \mathrm{cm}^{2}$ in conjunction with a laser spot size of $1.5 \mathrm{~mm}$ that was applied in a raster pattern with spot overlap as indicated in the schematics of Table 1. Differences in the LSP treatment of the samples pertain to the laser spot coverage densities as summarised in Table 1. In all cases the laser track lines were along the plate widths.

Table 1: Parametric variation of spot density in samples

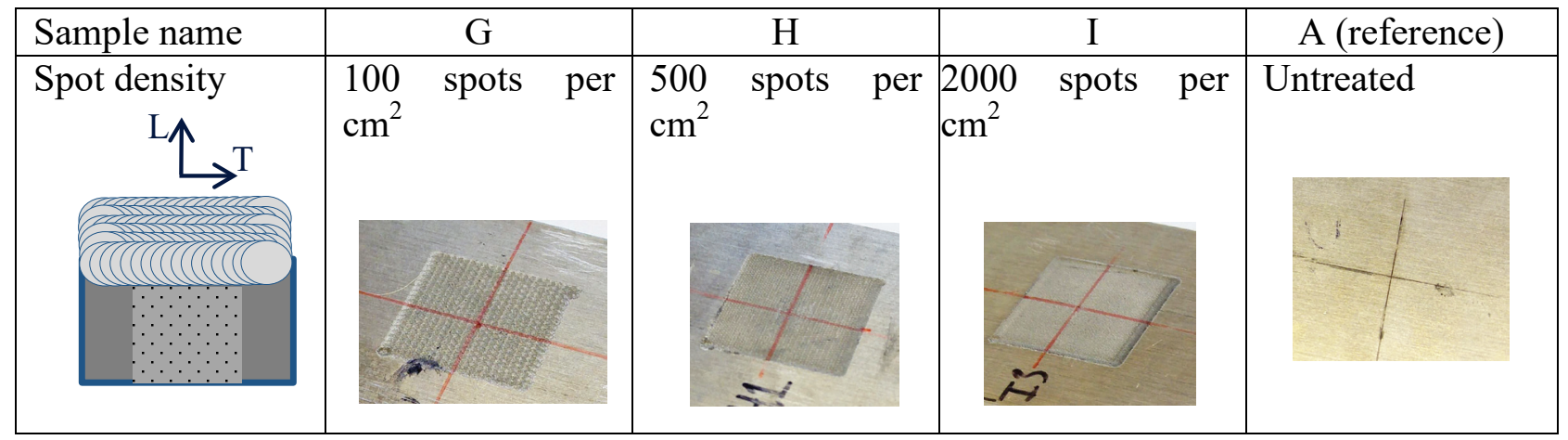

Principal sample (and strain) directions are defined in terms of the plate geometry as longitudinal (L) parallel to the long dimension of the plate which coincides with the rolling direction, transverse (T) across the plate length, and normal $(\mathrm{N})$, normal to the plate surface. The as-received parent material plate was used as stress reference that was investigated with the same setup and measurement protocol.

MPISI experimental procedure. MPISI is a constant wavelength diffraction instrument that delivers a $1.659 \AA$ wavelength neutron beam onto the instrument CoR when diffracting from the (331) plane of a silicon multiwafer single crystal monochromator at a fixed take-off angle of $83.5^{\circ}$. This places the diffraction angle from the (311) planes of aluminium at approximately $85.5^{\circ}$ as measured with the $2 \mathrm{D}$ neutron detector. A matchstick-shaped gauge volume was established with the primary beam aperture width set at $0.6 \mathrm{~mm}$ and $15 \mathrm{~mm}$ in height, with the secondary aperture width at $0.6 \mathrm{~mm}$ this rendered a nominal gauge volume of $5.4 \mathrm{~mm}^{3}$. Both apertures were positioned $20 \mathrm{~mm}$ from the CoR to minimize beam divergence and precisely aligned by means of through-thickness intensity scans from an aluminium pin located at the CoR. Fig. 2a-d shows the experimental setup and measuring directions of the samples. Alignment of the sample with respect to the neutron gauge volume was achieved by performing through-wall-thickness intensity scans from the back surfaces (opposite to the peened faces) of all samples and determining the surface position using the procedures described in [5]. Alignment accuracies are estimated to be better than $\pm 10 \mu \mathrm{m}$.

Strain measurements have been performed through the sample thickness in the central part of the LSP treatment patch as line scans by applying different measurement point densities. Close to the peened surfaces measurements were done in $0.2 \mathrm{~mm}$ steps, as overlapped gauge volumes, over the first millimeter to capture the near-surface peening effect, followed by step sizes of $0.5 \mathrm{~mm}$ to cover the remainder of the sample thickness. A total of 11 points (per strain direction) were thus measured through thickness. In order to compensate for spurious strains resulting from partially submerged gauge volumes at the sample surfaces, each sample was rotated $180^{\circ}$ in the horizontal plane and the depth-dependence of the strain component re-measured at corresponding positions to the first measurements. The $\mathrm{d}$-values at each corresponding position were averaged for the $0^{\circ}$ and $180^{\circ}$ 
orientations as described in [6]. Data acquisition times for all data points were $3600 \mathrm{~s}$ that rendered $\Delta d / d$ better than $10^{-4}$.

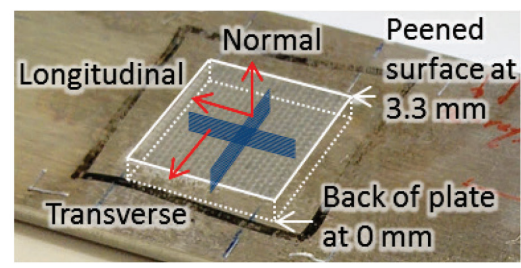

(a)

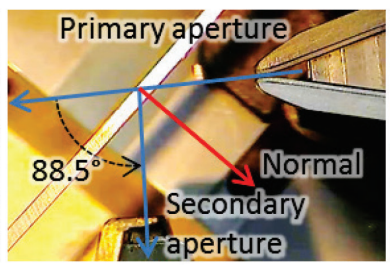

(b)

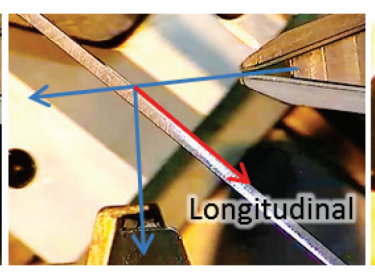

(c)

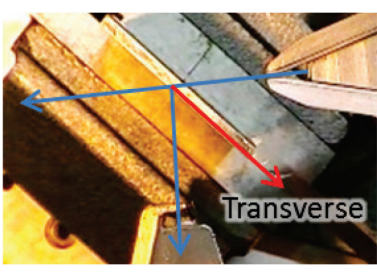

(d)

Fig.1: Schematic depictions of the orientations and through-thickness measurement regions (a). Photographs showing the sample setups on MPISI for the measurement of the three principal strain directions normal (b), longitudinal (c) and transverse (d). The longitudinal and transverse components were measured with the sample in transmission geometry whereas the normal component was measured in reflection geometry.

KOWARI experimental procedure. KOWARI is a constant wavelength diffraction instrument with a variable take-off angle. A take-off angle setting of $79^{\circ}$ delivered $1.76 \AA$ wavelength neutrons from the (400) refection of a silicon monochromator that put the (311) reflection of aluminium at $\sim 90^{\circ}$. A nominal gauge volume of $0.2 \times 15 \times 0.2 \mathrm{~mm}^{3}$ was established with the primary and secondary apertures positioned at $10 \mathrm{~mm}$ from the CoR to minimize the beam divergence.

The same instrument and sample setup procedures as used on MPISI were employed, though with faster measurement times. Through-thickness points were measured with regular $0.2 \mathrm{~mm}$ steps resulting in 16 overall points plus one additional point on the very surface. Data acquisition times of $1000 \mathrm{~s}$ for the T-component and $1500 \mathrm{~s}$ for the L-component were applied to compensate for texture related intensity variations in the plates, and $1200 \mathrm{~s}$ for the N-component. Acquisition times were increased by $50 \%$ for the first measurement position (closest to the peened surfaces) where data were taken with $1 / 2$ submerged gauges. This gave diffraction peak intensities to provide $\Delta d / d$ better than $10^{-}$ 4. At each depth, measurements were taken by stepwise translation of the sample parallel to the surface at each depth (equivalent to "oscillating the sample") over a distance of $2 \mathrm{~mm}$ in $0.5 \mathrm{~mm}$ steps to improve the grain statistics. This protocol is depicted in the measurement grid shown in Fig. 2a-c. The 5 data points at each depth were averaged in the data analysis.

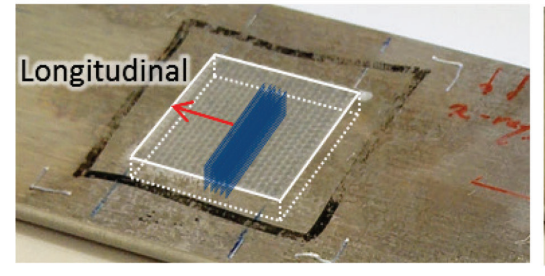

(a)

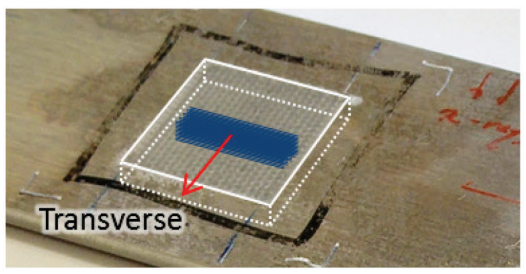

(b)

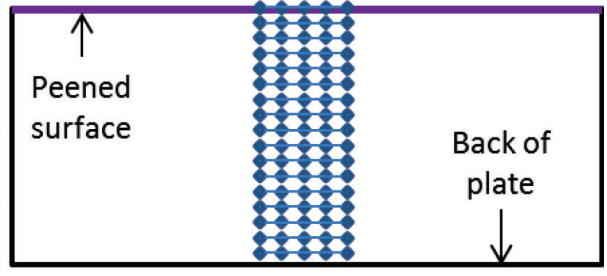

(c)

Fig. 2: Depiction of the measurement grid employed for the longitudinal (a) and transverse (b) component measurements on KOWARI. A cross sectional view of the in-plane and throughthickness measurement grid is given in (c).

\section{Results}

A reasonable assumption for thin plates is that the stress normal to the surface $\sigma_{\perp}$ is zero, from which the in-plane stress $\left(\sigma_{/ /}\right)$component can be determined by enforcing a bi-axial stress condition

$$
\sigma / /=\frac{1}{1 / 2 S_{2}(h k l)}\left(\frac{\mathrm{d} / /-\mathrm{d}}{\mathrm{d}_{0}}\right)
$$

where $1 / 2 S_{2}(\mathrm{hkl})$ is the corresponding diffraction elastic constant for the material and reflection, $d_{/ /}$ $\left(d_{L}\right.$ or $\left.d_{T}\right)$ and $d_{\perp}\left(d_{N}\right)$ respectively the in-plane and normal components of the lattice plane spacing at 
the corresponding depth positions. The values for the diffraction elastic constants of aluminium used are $S_{1}(311)=-5.158 \mathrm{TPa}^{-1}$ and $1 / 2 S_{2}(311)=19.574 \mathrm{TPa}^{-1}$ as determined from the single crystal elasticity constants using the Kröner approximation. Under the plane-stress approximation, the stressfree lattice plane spacing per point was calculated from

$$
\mathrm{d}_{0}=\left(\frac{1 / 2 S_{2}(h k l)+2 S_{1}(h k l)}{1 / 2 S_{2}(h k l)}\right) \mathrm{d}_{\mathrm{N}}-\frac{S_{1}(h k l)}{1 / 2 S_{2}(h k l)}\left(\mathrm{d}_{\mathrm{L}}+\mathrm{d}_{\mathrm{T}}\right)
$$

Stress results, with partial illumination corrections applied, are indicated in Fig. 3. In all curves the MPISI results are indicated as points only, in blue, whereas the data points for the KOWARI results are linked with lines (guides to the eye) in red. In the parent material significant differences are evident between the $\mathrm{L}$ and $\mathrm{T}$ stress components, especially at mid-plate thickness where distinctively large compressive stresses exist in the L-component (parallel to the rolling direction), that are balanced by slight tensile stresses at the surface regions. This is reminiscent of the deep (hot) rolling manufacture of the plate with consecutive cold rolling. The T-component does not reflect any large variation with thickness. A very good correlation is observed between the results determined with MPISI and KOWARI. These results on the reference sample were later used to obtain stresses purely associated with the peening action in the peened samples through point-by-point subtraction at each depth of the averaged L- and T-component stresses in the parent material from the stress values in the respective peened plates.
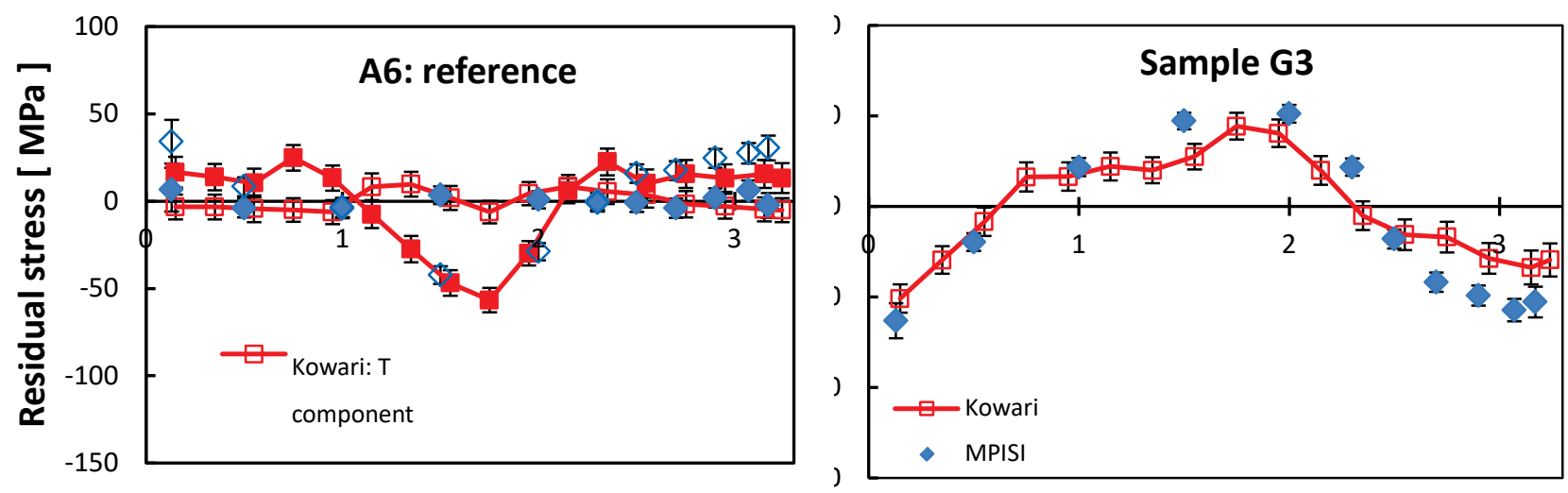

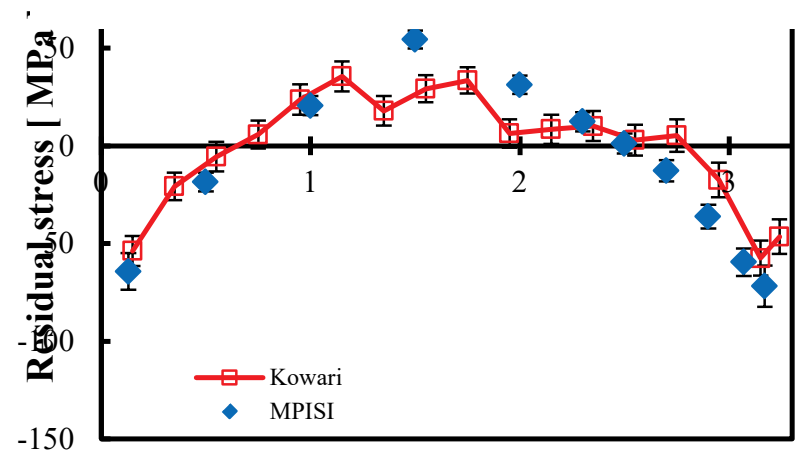

Position [ mm ]

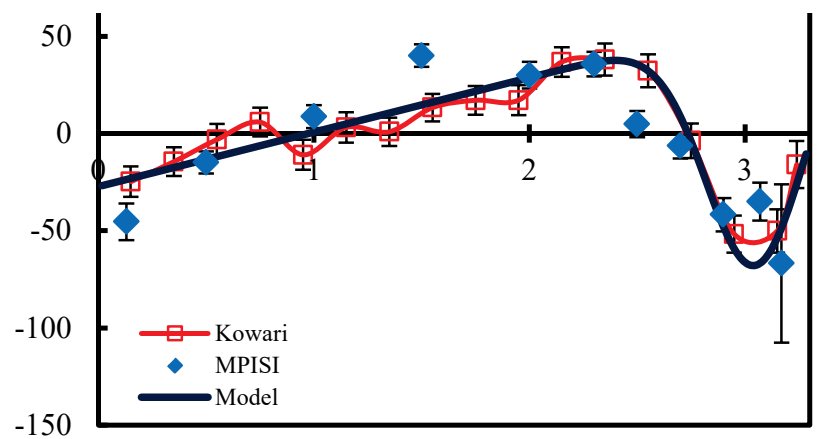

Position [ mm ]

Fig. 3: Depth-resolved in-plane residual stresses determined for the four samples of this study. Sample A serves as reference that represents the parent material. Results are depicted as the inplane stresses where the L-component refers to the stress along the plate rolling direction. Stresses depicted for the shock peened plates are the stresses purely due to the peening action. The peened surfaces are at $3.3 \mathrm{~mm}$. 
The calculated stress components $\mathrm{L}$ and $\mathrm{T}$ (not shown) in the peened plates have similar trends, but the magnitudes of the L-component are larger than the T-component, approximately $50 \%$ in sample $\mathrm{G}$ and $200 \%$ for samples $\mathrm{H}$ and I. This anisotropy is most likely related to the peening raster applied to cover the complete surface area that was done along lines over the plate widths and progressively translated along the plate length to accomplish the area coverage as shown in the schematic in Table 1. For the comparative characterization purposes, the stress fields are depicted by one profile, the in-plane stress. Hereafter, the individual longitudinal and transverse stress components were averaged and are reported in Fig. 3.

Sample I shows prominent reverse stress yielding at the peened surface and to a much lesser effect in sample H. Sample G shows a gradual stress gradient which becomes progressively more steep in samples H and I. In addition the results for sample I indicate the calculated peening stress distribution determined from the KOWARI data as input to an eigenstrain analysis [7]. Since the peen spot densities are progressively increased from sample $\mathrm{G}$ to $\mathrm{H}$ to $\mathrm{I}$, it is expected that the residual stress results should reflect a similar interrelationship. This is though not seen. This may be ascribed to the complicated and distributed stresses that already exist in the parent material, or over-peening with the LSP treatments. Measurements were repeated at two different parent material samples using KOWARI, but no significant differences were observed.

Another parameter that has been considered in the benchmarking is the full-width-at-halfmaximum of the Bragg peaks as a qualitative identifier of the plastic deformation regions. Results for the four samples are summarized in Fig. 4. In the parent material the peak widths are constant through thickness within the experimental errors. Sample G shows a slight increase in peak width as the peened surface is approached, whereas distinct peak broadening is observed in samples H and I. The peak broadening extends up to approximately $0.75 \mathrm{~mm}$ and $1.3 \mathrm{~mm}$ from the peened surface in samples $\mathrm{H}$ and I respectively. This indicates direct correlation between the peen spot density and the extent of the plastic yielding zone. Comparing the results between the two instruments reveals that the peak widths far from the peened regions are of similar resolution. In the peen-influenced region, the KOWARI results show better depth resolution between the different samples due to the smaller gauge volume employed.

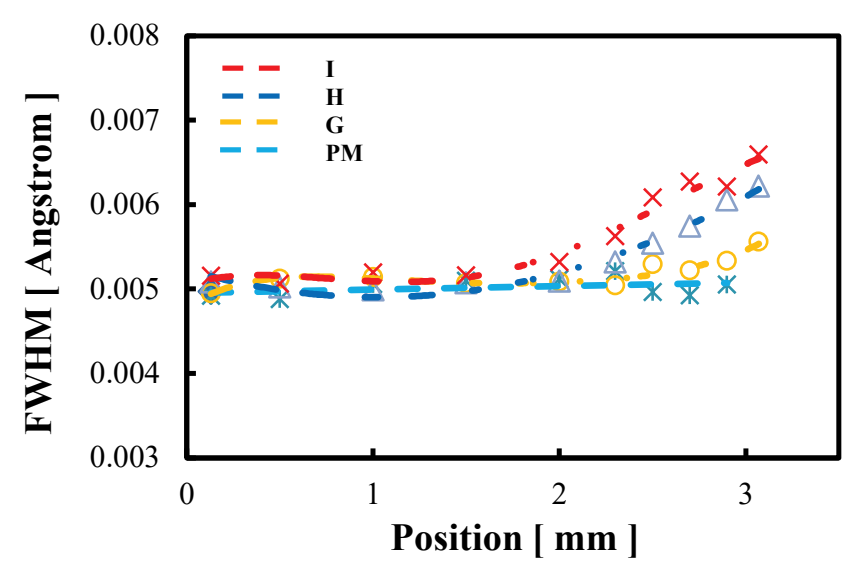

(a)

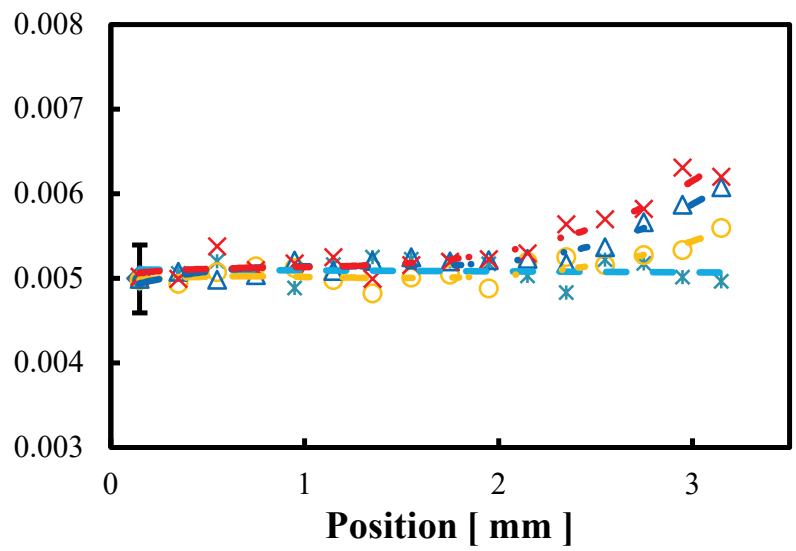

(b)

Fig. 4: Depth dependence of the FWHM for the four samples of this study. Curves have been determined from the diffraction data taken: (a) MPISI and (b) KOWARI. The solid lines indicate polynomial fits through the data points that serve as guides to the eye to indicate the trends.

\section{Discussion}

Notwithstanding the larger gauge volume used with the MPISI investigations and subsequent averaging of data over larger depth dimensions compared to KOWARI, essential features in the stress and FWHM profiles were captured. Due to the higher flux of KOWARI, nuances such as the reverse yielding profile in the near-to-surface region and discrimination of the plastically deformed regions could be resolved to greater detail. 
Using KOWARI results as a reference, analysis of the peak intensities versus stress values, uncertainties and spatial resolution obtained, either the data acquisition times on MPISI or the size of the gauge volume could be reduced by $50 \%$. For these high-resolution investigations the latter would be more advantageous and could be reduced to a gauge volume of $0.4 \times 15 \times 0.4 \mathrm{~mm}^{3}$ instead of 0.6 $\mathrm{x} 15 \times 0.6 \mathrm{~mm}^{3}$. In addition the depth resolution could be further improved by increasing the vertical dimension of the beam to $20 \mathrm{~mm}$ with a comparative reduction in the depth dimension. A similar "oscillation" measurement strategy should also be enforced to improve the grain statistics.

\section{Summary}

With the MPISI neutron strain scanner having been recently commissioned, the investigation formed part of a benchmarking study of MPISI against the KOWARI neutron strain scanner in the highspatial resolution regime. High-resolution depth-resolved measurements have been taken in an untreated and three LSP treated AA6056-T4 aluminium plates. Two in-plane components (longitudinal and transverse) as well as normal strain components were measured with a similar protocol and both attempting the best spatial resolution to provide cross-validation of the experimental results.

The latter investigation served to verify the experimental results obtained, as well as to yield some practical recommendations for optimizing high resolution measurement and to facilitate experimental unraveling of the residual stresses close to the peened surfaces. Overall, very good correlation is observed between the MPISI and KOWARI investigations.

\section{Acknowledgments}

AM Venter acknowledges financial support from Necsa SOC Limited and the use of the neutron strain scanner instruments MPISI and KOWARI under ANSTO proposal P4639. D Glaser is thanked for providing the LSP samples used in these studies.

\section{References}

[1] VAMAS report No 38 'Neutron diffraction measurements of residual stress in a shrink-fit ring and plug', G.A. Webster (Ed.), NPL, Teddington, UK, Jan 2000.

[2] K. Ding and Y Lin, Laser shock peening: Performance and process simulation, Woodhead Publishing, 2006.

[4] Y. Sano et al., Improvement in fatigue performance of friction stir welded A6061-T6 aluminium alloy by laser peening without coating, Materials \& Design 36 (2012) 809-814.

[3] Kirstein, O., Luzin, V., and Garbe, U., 2009, The Strain-Scanning Diffractometer Kowari: Neutron News, v. 20, no. 4, p. 34-36.

[5] P.C. Brand, H.J. Prask, New methods for the alignment of instrumentation for residual-stress measurements by means of neutron diffraction, J. Appl. Cryst. 27 (1994) 164-176.

[6] M.T. Hutchings, P.J. Withers, T.M. Holden, T. Lorentzen, Introduction to the Characterization of Residual Stress by Neutron Diffraction, Taylor and Francis, London, 2005.

[7] A. M. Korsunsky, 2005, On the modelling of residual stresses due to surface peening using eigenstrain distributions: Journal of Strain Analysis for Engineering Design, v. 40, no. 8 (2005) p. 817-824. 